\title{
Reproductive immunology research: a tight interaction between diverse scientific and clinical disciplines including immunology, obstetrics, hematology, and endocrinology
}

\author{
Stavros Giaglis ${ }^{1,2}{ }^{*}$ and Sinuhe Hahn ${ }^{1}$ \\ 1 Department of Biomedicine, University Hospital Basel, Basel, Switzerland \\ ${ }^{2}$ Department of Rheumatology, Kantonsspital Aarau, Aarau, Switzerland \\ *Correspondence: stavros.giaglis@unibas.ch \\ Edited and reviewed by: \\ Pietro Ghezzi, Brighton and Sussex Medical School, UK
}

Keywords: reproductive immunology, immunology, obstetrics, hematology, endocrinology

The unique immunological riddle of human gestation involves a broad variety of cells and molecules playing evidently important roles (1). The reproductive and immune systems are morphologically diffused, and their modus operandi involves widely distributed signals in response to the given environmental input, while both systems must interact to obtain their normal functionality (2). Furthermore, dysregulation of physiological interactions between the two systems can lead to severe pregnancy-related disorders or complications, such as fetal loss, preterm labor, preeclampsia (PE), and poor fetal development (3). On the other hand, by ameliorating autoimmune conditions such as multiple sclerosis and rheumatoid arthritis, while other conditions such as systemic lupus are deteriorated, pregnancy as a condition may provide a unique insight into novel immunomodulatory strategies $(4,5)$.

The scientific focus on reproductive-immune research has historically provided substantial understanding of the interface between these two physiological systems. With such recent progress in the field, we felt that it is valuable and well-timed to review the broad variety of the relevant physiologic and pathologic aspects - from menstruation to fertilization and implantation, and from placentation and pregnancy per se to the post partum condition - in which the immune system takes part.

As editors, we are delighted by the keen response of 15 groups of scientists from 4 different continents, which kindly contributed their unique expertise to our effort to recap, value, and extend our insights concerning the field of reproductive immunology. We sincerely hope that the present eBook will succeed to share with the reader the broad and vivid discussion between the authors and editors.

Schumacher et al. (6) explore the interplay between the endocrine and immune systems during gestation, with focus on progesterone, estradiol, and human chorionic gonadotropin. Pregnancy hormones are critically for the successful establishment, maintenance, and completion of pregnancy. They suppress detrimental maternal alloresponses while promoting tolerance pathways through the antigen-presenting capacity of DCs, monocytes, and macrophages as well as the blockage of NK, T, and B cells. These findings highlight the importance of endocrine factors for tolerance induction during pregnancy.

Hsu and Nanan (7) discuss the recent advances in the complex crosstalk between the innate and adaptive immune system during human pregnancy and PE. They present many lines of evidence supporting an immunological origin to $\mathrm{PE}$, implicating decidual NK cells and APC DCs and macrophages as major players in the regulation of vascular remodeling and trophoblast invasion. On the other hand, within the adaptive immune system, Foxp $3^{+}$ Tregs and $\mathrm{CD} 4^{+} \mathrm{HLA}_{-\mathrm{G}^{+}}$suppressor T-cells seem to be essential for guaranteeing immune tolerance.

Getting deeper into the mechanisms of placental pathology, Faas et al. (8) focus on the role of monocytes and macrophages in pregnancy and PE. Given the generalized activation of the acute inflammatory response, monocytes may play a central role in this reaction, since they are short-lived cells that mature in the circulation and transmigrate into affected tissues upon an inflammatory stimulus, developing into macrophages. Macrophages in turn are abundant in the endometrium and play a crucial part in implantation and placentation. In PE, these macrophages appear to be activated and in larger numbers.

In the same context, Ruocco et al. (9) provide insight concerning the role of Tregs in pregnancy reassessing the original concept of "suppressor T-cells" in pregnancy, putting it in a historical perspective, and highlighting the main data revising the concept of Tregs in gestation. Moreover, they focus to the most important questions in the field, such as Treg antigen specificity, Treg subsets, the functional crosstalk of Tregs with NK and DCs.

Joerger-Messerli et al. in Basel (10) observed that the inflammatory reaction in monocytes is initiated by the interaction of syncytiotrophoblast microparticles (STBM) with TLRs, which in turn signal through NF- $\mathrm{KB}$ to mediate the transcription of proinflammatory mediators. Since pregnancy is accompanied by a mild systemic inflammatory response, they show that in vitro generated STBM from normal placentas stimulate monocytes. Furthermore, STBM derived from PE placentas up-regulated CD54 expression, and stimulated IL- 6 and IL- 8 secretion in a dosedependent manner, which was impaired in the presence of TLR signaling inhibitors or when blocking NF-кB activation.

Fettke et al. (11) examine B cell involvement in the immune response against paternal antigens and tolerance mechanisms. Such pleiotropic cells play a considerable role by secreting immunomodulatory IL-10, while they can harm pregnancy due to their capacity to produce autoantibodies. New evidence in mouse models suggests that IL-10 producing B cells (B10), contribute 
in maintaining tolerance, fighting danger signals at the fetalmaternal interface. In human pregnancies, B10 cells increase with onset but not in case of spontaneous abortions, suppressing TNF- $\alpha$ production by $\mathrm{T}$-cells.

A review on the unique neonatal NK cell population and its role in gestational autoimmunity is provided by Rival et al. (12) from Kenneth Tung's group. Maternal autoantibodies can trigger autoimmune ovarian disease (AOD) in the progeny of women with SLE or Sjogren's syndrome. The pathogenic effect of autoantibody exposure is investigated in mice, in which immune complexes are formed in adult and neonatal ovaries, but a specific Ab-species triggers severe AOD only in young mice. Propensity to AOD is due to the uniquely hyper-responsive neonatal NK cells. Resistance to AOD in older mice results from specific NK cells that regulate effector NK cells and Tregs. Activated by ovarian immune complexes, NKs migrate to lymphoid organs where priming occurs. These insights uncover new properties of the neonatal innate and adaptive responses, lethality of premature infant infection, and novel neonatal antiviral vaccine design.

Woidacki et al. (13) summarize the existing knowledge concerning the course of pregnancy in women affected by mast cell (MC) mediated or associated disorders. While physiological numbers of MCs influence positively the outcome of pregnancy, uncontrolled augmentations in quantity and activation can lead to dangerous complications. Women with the desire of getting pregnant and diagnosed with MC mediated disorders - urticaria, mastocytosis, or MC-related chronic inflammatory diseases - may benefit from specialized medical support to ensure a positive pregnancy outcome.

Than et al. (14) provide evidence concerning the protective role of placental protein 13 (PP13), an immunoregulatory galectin. Three of the five human galectins are expressed in the placenta, and galectin-13 (PP13) is predominantly expressed by the syncytiotrophoblast and released from the placenta into the circulation. Its ability to induce apoptosis of T-cells in vitro and to kill T-cells and macrophages in the maternal decidua, suggests important immune functions. Indeed, LGALS13 mutations and decreased placental expression of PP13 and its low concentrations during first trimester are associated with elevated risk of PE. PP13 turned to be a good early biomarker to assess maternal risk for the subsequent development of pregnancy complications, which might enable its potential in directing patient management.

Sedlmayr et al. (15) highlight the role of tryptophan catabolism in the placenta, focusing mainly on the role of indoleamine 2,3-dioxygenase-1 (IDO1), one of three enzymes involved in the tryptophan degradation pathway. IDO1 has been implicated in regulation of feto-maternal tolerance in the mouse. Depletion of tryptophan mediates immunoregulation and antimicrobial functions. In addition to the decidual glandular epithelium, IDO1 is localized in the vascular endothelium of the chorion and the endothelium of the decidual spiral arteries. Possible consequences of tryptophan catabolism in the endothelium are relaxation of the placental vasotonus, contributing to placental perfusion and growth of both placenta and fetus.

Chatterjee et al. (16) from the Mitchell lab examine how immune cells that produce IL-4 and IL-10 are regulated throughout pregnancy and the effects of reduced IL-4 and IL10 signaling on fetal and maternal physiology. The resolution of inflammation plays an important role throughout pregnancy and is largely mediated by immune cells producing IL- 4 and IL- 10 . The temporal and spatial aspects of reducing inflammation during pregnancy are thoroughly discussed.

Perez-Sepulveda et al. (17) discuss the involvement of the innate immune system in the establishment of an environment that favors pregnancy and possible alterations related to the development of PE. Since normal pregnancy is considered as a Th2 immunological state, PE has been classically described as a Th1/Th2 imbalance; recent studies have expanded the Th1/Th2 into a Th1/Th2/Th17 and regulatory T-cells paradigm and where DCs could have a crucial role.

An insight into the impact of bacterial infections, such as Helicobacter pylori (HP), in PE is delivered by Tersigni et al. (18). Since the primary trigger of $\mathrm{PE}$ is unknown, a hypothesis concerning the disease onset is triggering by infectious agents. Consistently, higher seroprevalence of HP infection is evident in women with $\mathrm{PE}$. As trophoblast invasion is a crucial step in implantation and placental development, the proposed infection-induced autoimmune mechanism, interfering negatively with the fetal side of the developing placenta, may explain the higher seropositivity for HP infection PE cases.

Nilsson et al. (19) discuss the role of the human HLA-Ib protein (HLA-G) in the regulation of the immunological crosstalk during conception and pregnancy: from genetics to physiological effects, from pregnancy and pregnancy complications to a short discussion on future possible means of preventative measures and therapies. As HLA-G expression is limited to gestation, it is proposed as a key player in the maintenance of immunological tolerance. HLA-G might be involved in immune processes even before conception, since HLA-G is detected in nonpregnant women genital tract and blood, in men's seminal fluid, and in the pre-implanted embryo. Therefore, a combined contribution from the mother, the father, and the embryo/fetus is important.

The series of articles included in the present collection is completed with a commentary by Khoury et al. (20) on the promising potential of menstrual stem cells for antenatal diagnosis and cell therapy. Menstrual-derived stem cells (MenSCs) are a new source of MSC isolated from the menstrual fluid. Currently, there is a growing interest in their clinical potential due to their multipotency, high proliferative capacity, and facile way to obtain non-invasively. This review details their distinctive biological properties regarding immunophenotype and function, proliferation/differentiation potential, and paracrine effects. Their possible role in antenatal diagnosis is also discussed.

\section{ACKNOWLEDGMENTS}

We cordially thank all the participants and the numerous committed reviewers for their major support, substantial effort, and great enthusiasm. We hope that this special group of contributors and others drawn to this field will remain highly productive in the future and we are sincerely looking forward for our readers to profoundly benefit from new exciting insights and fruitful collaborations. 


\section{REFERENCES}

1. Scott JS. Immunological diseases and pregnancy. Br Med J (1966) 1:1559-67. doi:10.1136/bmj.1.5503.1559

2. Munoz-Suano A, Hamilton AB, Betz AG. Gimme shelter: the immune system during pregnancy. Immunol Rev (2011) 241:20-38. doi:10.1111/j.1600-065X. 2011.01002.x

3. Arck PC, Hecher K. Fetomaternal immune cross-talk and its consequences for maternal and offspring's health. Nat Med (2013) 19:548-56. doi:10.1038/nm. 3160

4. Bulmer JN. Immune aspects of pathology of the placental bed contributing to pregnancy pathology. Baillieres Clin Obstet Gynaecol (1992) 6:461-88. doi:10.1016/S0950-3552(05)80006-9

5. Hahn S, Giaglis S, Hoesli I, Hasler P. Neutrophil NETs in reproduction: from infertility to preeclampsia and the possibility of fetal loss. Front Immunol (2012) 3:362. doi:10.3389/fimmu.2012.00362

6. Schumacher A, Costa SD, Zenclussen AC. Endocrine factors modulating immune responses in pregnancy. Front Immunol (2014) 5:196. doi:10.3389/ fimmu.2014.00196

7. Hsu $P, N a n a n ~ R K$. Innate and adaptive immune interactions at the fetal-maternal interface in healthy human pregnancy and pre-eclampsia. Front Immunol (2014) 5:125. doi:10.3389/fimmu.2014.00125

8. Faas MM, Spaans F, De Vos P. Monocytes and macrophages in pregnancy and pre-eclampsia. Front Immunol (2014) 5:298. doi:10.3389/fimmu.2014.00298

9. Ruocco MG, Chaouat G, Florez L, Bensussan A, Klatzmann D. Regulatory Tcells in pregnancy: historical perspective, state of the art, and burning questions. Front Immunol (2014) 5:389. doi:10.3389/fimmu.2014.00389

10. Joerger-Messerli MS, Hoesli IM, Rusterholz C, Lapaire O. Stimulation of monocytes by placental microparticles involves toll-like receptors and nuclear factor kappa-light-chain-enhancer of activated B cells. Front Immunol (2014) 5:173. doi:10.3389/fimmu.2014.00173

11. Fettke F, Schumacher A, Costa SD, Zenclussen AC. B cells: the old new players in reproductive immunology. Front Immunol (2014) 5:285. doi:10.3389/fimmu. 2014.00285

12. Rival C, Setiady Y, Samy ET, Harakal J, Tung KS. The unique neonatal NK cells: a critical component required for neonatal autoimmune disease induction by maternal autoantibody. Front Immunol (2014) 5:242. doi:10.3389/fimmu.2014. 00242

13. Woidacki K, Zenclussen AC, Siebenhaar F. Mast cell-mediated and associated disorders in pregnancy: a risky game with an uncertain outcome? Front Immunol (2014) 5:231. doi:10.3389/fimmu.2014.00231
14. Than NG, Balogh A, Romero R, Karpati E, Erez O, Szilagyi A, et al. Placental protein 13 (PP13) - a placental immunoregulatory galectin protecting pregnancy. Front Immunol (2014) 5:348. doi:10.3389/fimmu.2014.00348

15. Sedlmayr P, Blaschitz A, Stocker R. The role of placental tryptophan catabolism. Front Immunol (2014) 5:230. doi:10.3389/fimmu.2014.00230

16. Chatterjee P, Chiasson VL, Bounds KR, Mitchell BM. Regulation of the antiinflammatory cytokines interleukin-4 and interleukin-10 during pregnancy. Front Immunol (2014) 5:253. doi:10.3389/fimmu.2014.00253

17. Perez-Sepulveda A, Torres MJ, Khoury M, Illanes SE. Innate immune system and preeclampsia. Front Immunol (2014) 5:244. doi:10.3389/fimmu.2014.00244

18. Tersigni C, Franceschi F, Todros T, Cardaropoli S, Scambia G, Di Simone N. Insights into the role of Helicobacter pylori infection in preeclampsia: from the bench to the bedside. Front Immunol (2014) 5:484. doi:10.3389/fimmu.2014. 00484

19. Lynge Nilsson L, Djurisic S, Hviid TV. Controlling the immunological crosstalk during conception and pregnancy: HLA-G in reproduction. Front Immunol (2014) 5:198. doi:10.3389/fimmu.2014.00198

20. Khoury M,Alcayaga-Miranda F, Illanes SE, Figueroa FE. The promising potential of menstrual stem cells for antenatal diagnosis and cell therapy. Front Immunol (2014) 5:205. doi:10.3389/fimmu.2014.00205

Conflict of Interest Statement: The authors declare that the research was conducted in the absence of any commercial or financial relationships that could be construed as a potential conflict of interest.

Received: 12 December 2014; accepted: 07 January 2015; published online: 23 January 2015.

Citation: Giaglis S and Hahn S (2015) Reproductive immunology research: a tight interaction between diverse scientific and clinical disciplines including immunology, obstetrics, hematology, and endocrinology. Front. Immunol. 6:10. doi: 10.3389/fimmu.2015.00010

This article was submitted to Inflammation, a section of the journal Frontiers in Immunology.

Copyright (C) 2015 Giaglis and Hahn. This is an open-access article distributed under the terms of the Creative Commons Attribution License (CC BY). The use, distribution or reproduction in other forums is permitted, provided the original author (s) or licensor are credited and that the original publication in this journal is cited, in accordance with accepted academic practice. No use, distribution or reproduction is permitted which does not comply with these terms. 